\title{
Packet Loss Effects in Passive Telepresence Systems
}

\author{
Sandra Hirche and Martin Buss
}

\begin{abstract}
This paper focuses on the effects of packet loss in passive bilateral telepresence systems with force feedback. The substantial impact of packet loss and subsequently applied packet processing algorithms on the energy balance and thereby stability and performance of passive telepresence systems is studied. As an example for a class of non-energy preserving packet processing algorithms, the Hold Last Sample (HLS) strategy, is shown to generate energy. A sufficient condition for the non-passivity of HLS is derived and passifying compensation is discussed.
\end{abstract}

\section{INTRODUCTION}

Telepresence systems enable the human operator to perceive and act in a remote environment. For an overview and application potential (telemanufacturing, telemaintenance, telesurgery, rescue, etc.) of telepresence see e.g. [1]. Challenges in telepresence are time delays in the range $20 . .300 \mathrm{~ms}$ round trip, which can be time-varying. Another important isssue is packet loss in packet switched communication networks like e.g. the Internet.

The architecture of a telepresence system is depicted in Fig. 1. The human operator manipulates the force feedback capable Human System Interface (HSI) thereby commanding the executing robot (teleoperator). While the teleoperator interacts with the usually unknown remote environment the sensor data (visual, auditory, haptic) are fed back and displayed to the operator. Ideally, the human operator feels directly connected to the remote environment, which is called transparency. Considering the visual and auditory feedback as state-of-the-art-multimedia, this paper focuses on the haptic feedback subsystem. For the communication of the command signals and sensor data we assume a digital packet switched communication network.

Telepresence systems belong to the class of Networked Control System (NCS). In terms of a NCS the human operator together with the HSI acts as controller providing control signals to the remote system consisting of teleoperator and environment. The teleoperator can be interpreted as an actuator equipped with sensors. By communicating back the sensor data a global control loop is closed over the communication network. Subsequently, we discuss issues important for force reflecting telepresence systems and note that these results can readily be carried over to the standard NCS problem (subject to further investigations).

The stability and performance of telepresence systems is highly affected by the quality of communication. In an unreliable packet switched network, as e.g. the Internet, variable network traffic possibly resulting in congestion

Both authors' affiliation: Institute of Automatic Control Engineering, Technische Universität München, D-80290 München, Germany http: //www.lsr.ei.tum.de,

Sandra.Hirche@ei.tum.de

Martin.Buss@ei.tum.de determines the time delay, its variation, and packet loss from the network point of view. The effective end-to-end delay and the packet loss seen by the telepresence system are determined by the packet processing algorithms. Packet processing in this context stands for the treatment of early, late, and lost packets.

In [2] the passivity concept with resulting stabilizing control methods using the scattering transformation is proposed to render a haptic telepresence system with arbitrary but constant delay stable. An extension for the time varying, continuous time case can be found in [3], [4]. In [5] underlying discrete nature of Internet-like networks is taken into account, a port-Hamiltonian system model for the telepresence system with communication network is derived.

Packet loss or increasing time delay may result in a buffer under-run at the receiver side. In [6] two different compensation schemes for the treatment of the resulting vacant sampling instances are proposed: keeping the old control (Hold Last Sample) or generating new control with estimated data. Both techniques have been applied in NCS. Packet loss compensation by holding the last received sample has also been employed in [7]. Estimation techniques for NCS based on recursive and Kalman filters have been suggested in [8], [9]. All these compensation methods target at the signal reconstruction without energy conservation considerations. In [10] a passivity preserving communication signal management has been proposed. This algorithm induces additional delay through packet buffering at the receiver side.

In this paper packet loss compensation methods are investigated. We will show that the Hold Last Sample algorithm potentially generates energy, hence does not preserve passivity. In consequence stability of the telepresence system cannot be guaranteed. Two passivation methods that do not introduce additional delay are proposed.

The paper is organized as follows: section II introduces passivity based stabilization methods; section III discusses packet loss in unreliable networks in combination with packet processing algorithms. In section IV a sufficient condition for the non-passivity of the Hold Last Sample strategy is derived; a passifying approach is discussed in section V.

\section{Telepresence Systems with Time Delay}

A haptic telepresence system basically consists of a force feedback capable HSI (variables indexed ${ }_{h}$ ) and the teleoperator (index ${ }_{t}$ ) interacting with an usually unknown remote environment (index $e_{e}$ ) as shown in Fig. 1. In bilateral telepresence the human manipulates the HSI applying the 


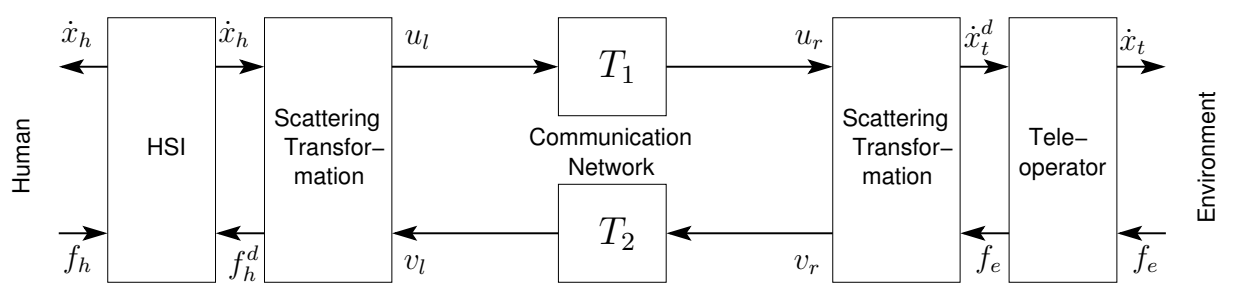

Fig. 1. Telepresence system architecture with communication two-port.

force $f_{h}$. Based on stability arguments in the standard architecture the HSI velocity $\dot{x}_{h}$ is communicated to the teleoperator where a local velocity control loop ensures the tracking of the desired teleoperator velocity $\dot{x}_{t}^{d}\left({ }^{d}\right.$ denotes desired). The force $f_{e}$ sensed at the remote site, resulting from the interaction with the environment, is transmitted back to the HSI serving as reference signal $f_{h}^{d}$ for local force control. The HSI and the teleoperator are modeled as mass damper systems, with the appropriate control inputs, the human force input $f_{h}$ at the HSI

$$
m_{h} \ddot{x}+b_{h} \dot{x}_{t}=\tau_{h}+f_{h},
$$

and the environment force input $f_{e}$ at the teleoperator

$$
\begin{aligned}
m_{t} \ddot{x}+b_{t} \dot{x}_{t} & =\tau_{t}-f_{e} \\
f_{e} & =b_{e} \dot{x}_{e}+k_{e} x_{e},
\end{aligned}
$$

with $\ddot{x}$ representing the acceleration, $m$ the mass, $b$ the damping, $k$ the spring coefficient and $\tau$ the control input from some appropriate chosen controller. We assume the teleoperator to be attached to the environment, hence $\dot{x}_{e}=\dot{x}_{t}$ and $x_{e}=x_{t}$.

The HSI and the teleoperator are connected through a communication network with time delay closing a global control loop via the human operator. Without further control measures the closed loop system is unstable.

\section{A. The Passivity Approach}

A common approach to analyze and synthesize telepresence system architectures with time delay is the passivity concept providing a sufficient condition for stability. A complex system of interconnected network elements ( $n$ ports) is passive, and implicitly stable, if each of the subsystems is passive. A passive element is one for which the property

$$
E_{\text {in }}(t)=\int_{0}^{t} P_{\text {in }} \mathrm{d} \tau=\int_{0}^{t} u^{T} y \mathrm{~d} \tau \geq-E_{\text {in }}(0) \quad \forall t>0
$$

holds, with $P_{\text {in }}$ and $E_{\text {in }}(t)$ denoting the power and the energy dissipated/stored in the system, $E_{\text {in }}(0)$ is the energy storage at $t=0$. The system input and the output vector are denoted by $u$ and $y$ respectively. In classical telepresence architectures, as proposed in [2], the appropriately locally controlled HSI and teleoperator exchange velocity and force signals, as the mapping from velocity to force is generally passive, hence the teleoperator and the HSI are passive subsystems. The environment is considered passive and it is assumed that the (possibly so trained) human operator behaves in a cooperative way rendering the overall system passive and therewith stable.

\section{B. Constant Delay}

In the following we consider a haptic telepresence system with constant time delay and without packet loss.

The bidirectional communication channel can then be modeled as a time delaying two-port with time delays $T_{1}$, $T_{2}$ in the forward and the backward path, respectively, see Fig. 1. As shown in [2] the communication two-port is not passive. The scattering transformation first applied to telepresence systems in [2] and then in [11] passivates the communication two-port for constant delays with the wave variable transformation equations

$$
\begin{aligned}
u_{l} & =\frac{1}{\sqrt{2 b}}\left(f_{h}^{d}+b \dot{x}_{h}\right) ; & u_{r} & =\frac{1}{\sqrt{2 b}}\left(f_{e}+b \dot{x}_{t}^{d}\right) \\
v_{l} & =\frac{1}{\sqrt{2 b}}\left(f_{h}^{d}-b \dot{x}_{h}\right) ; & v_{r} & =\frac{1}{\sqrt{2 b}}\left(f_{e}-b \dot{x}_{t}^{d}\right) .
\end{aligned}
$$

with $u_{r}(t)=u_{l}\left(t-T_{1}\right)$ and $v_{l}(t)=v_{r}\left(t-T_{2}\right)$. Using the notation of (1) with the input vector $u^{T}=\left[\dot{x}_{h}-\dot{x}_{t}^{d}\right]$, the output vector $y^{T}=\left[f_{h}^{d} f_{e}\right]$ and given zero energy storage at $t=0$ the communication two-port stores the energy

$$
\int_{0}^{t} P_{i n} \mathrm{~d} \tau=\int_{0}^{t}\left(\dot{x}_{h} f_{h}^{d}-\dot{x}_{t}^{d} f_{e}\right) \mathrm{d} \tau \geq 0 \quad \forall t .
$$

If the delay varies over time $T_{1}=T_{1}(t), T_{2}=T_{2}(t)$ the transmitted signals are distorted. Increasing time delay renders the communication two-port non-passive; passivity is preserved in case of decreasing delay. The extended method from [3] introduces time varying gains to shape the energy output of the communication two-port and thereby passifies the communication line. This method proposed for the continuous time case can not directly be applied to telepresence systems over packet switched networks due to its packet oriented nature.

\section{PACKet Loss in UnReliable Networks}

We now consider a sampled data system for HSI and teleoperator control and a multi-path packet switched network with time-varying delay and packet loss for communication. We assume that the sender as well as the receiver side operate with the same sampling time $T_{A}$. Without loss of generality we assume that each data packet contains one sample $u_{l}(k)\left(v_{r}(k)\right.$ for the backward path) representing the 
output of discrete time scattering transformation [13], see Fig. 2. Hence packetizing is not considered. Here $u_{l}(k)$ indicates the value of the discrete variable $u_{l}(t)$ corresponding to the interval $t \in\left[k T_{A},(k+1) T_{A}\right]$. The data packets are put on the outgoing link in equal fixed time intervals $T_{A}$.

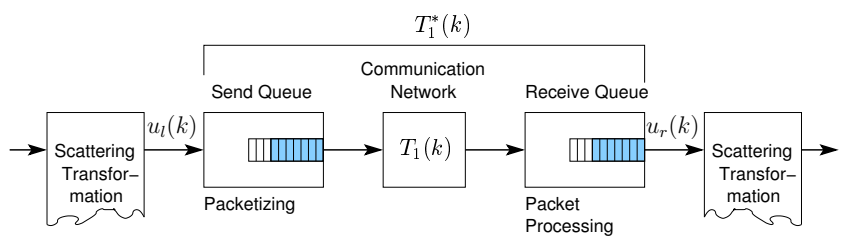

Fig. 2. Communication network with underlying packet nature and processing algorithms.

\section{A. Packet Loss due to Congestion}

In a congested network data packets are discarded at congested receive queues of intermittent routers resulting in packet loss. The underlying protocol has an effect on the packet loss and the delay. TCP like protocols with a resend algorithm detect this packet loss and resend the data resulting in increased delay for the affected packet. Furthermore, the congestion control of TCP, i.e. the sending rate adaption known as window behavior may result in increasing delay. Unreliable protocols without implicite congestion control like UDP do not recover packet loss. In realtime applications as telepresence systems the UDP protocol is preferred over the TCP with its inferior realtime characteristics.

\section{B. Packet Loss as a Result of Time-Varying Delay}

During transmission over the network each of the data packets experiences a time-varying link delay of $T_{1}(k)$ ( $T_{2}(k)$ in the backward path), depending on the load and the underlying protocol. In dynamically routed multi-path networks as e.g. the Internet the packets may arrive at the receiving queue in permutated order as result of decreasing delay or the underlying protocol. Increasing delay may result in empty sampling instances at the receiver side. In Fig. 3 the sent and received packets are depicted with their arriving order showing an empty sampling instance at $k=6$ and permutated arrival orders of the packets \#2 and \#3 at $k=7,8$.

The packet processing algorithm on the receiver side handles the permutated order arrivals and empty sampling instances. Two extremes of possible approaches with respect to their effect to end-to-delay and loss are discussed here. In [14] packet buffering and reordering is proposed. The buffer is designed such that each packet experiences the maximum communication delay, see Fig. 3 a). This approach has the advantage of not introducing additional loss, but results in possibly high end-to-end delay $T_{1}^{*}$. In the UseFreshest-Sample (UFS) algorithm no packet buffering is considered. Older packets are discarded in favor of younger packets, see Fig. 3 b), where packet \#2 is discarded. As a result no additional delay, but additional packet loss is

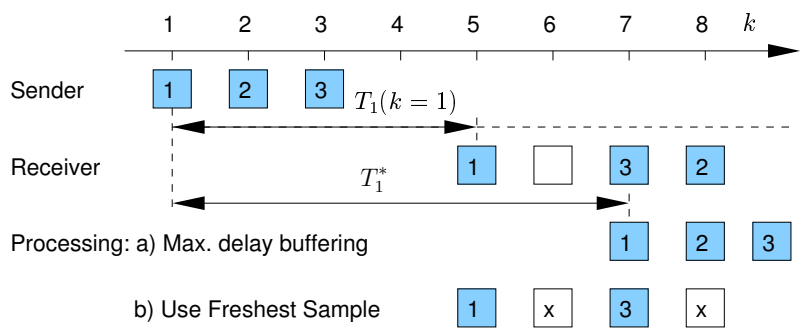

Fig. 3. Effect of varying delay in packet switched networks and processing algorithms, signal values of the sampling instances marked with ' $x$ ' need to be estimated.

imposed. Between these two extremes other algorithms with different levels of trade-offs between additional loss and additional delay can be considered. The optimum with respect to the transparency of the telepresence system is subject to further investigations.

In summary in packet switched networks depending on the packet processing algorithm time-varying delay may result in additional packet loss and/or additional but constant delay. The packet loss due to network congestion and processing induced loss sum up to the overall packet loss. In the following we will show that the treatment of empty sampling instances has a significant effect on passivity.

\section{Hold Last SAmple - Effect on PAssivity}

In case of a short term signal outage due to packet loss either due to network congestion or processing the missing data can be estimated. One can imagine a whole class of algorithms that use the information of previous signal behavior, but do not consider the energy balance. A very simple strategy belonging to this class of algorithms is the Hold-Last-Sample (HLS) strategy. In case that there is no more data packet in the receive queue, the last sample of the actual packet is held until the next younger packet arrives. In terms of the time delay this means, that with every time step $T_{A}$ the overall delay increases by one time step $T_{A}$. Comparing with the continuous time case implicitly there is the possibility of energy generation due to the HLS algorithm as shown in the following.

\section{A. Non-Passivity of HLS}

A sufficient condition for the non-passivity of the HLS algorithm is now derived. Without loss of generality we present our considerations in continuous time; all results can as well be stated for the discrete time case if the passivity integral (1) is expressed as a sum. For clarity the subsequent discussion refers to the forward path only, but equally applies to the backward path.

Theorem 1: Under the assumption of a single HLS in the forward path in the time interval $\left[t_{1}, t_{2}\right]$ with $t_{1} \geq T_{1}$, the communication two-port with constant delay and scattering transformation is non-passive if the inequality

$$
-\left(t_{2}-t_{1}\right) \cdot u_{l}^{2}\left(t_{1}-T_{1}\right)+\int_{t_{1}}^{t_{2}} u_{l}^{2}\left(\tau-T_{1}\right) \mathrm{d} \tau<0
$$


holds.

Proof: The idea of the proof is to compare the energy in the communication two-port without HLS and with HLS, $E_{i n}(t)$ and $E_{i n}^{H L S}(t)$. As the energy balance is only affected during the HLS interval the energy difference of communications with HLS and no loss yields

$$
E_{i n}^{H L S}(t)-E_{i n}(t)=\int_{t_{1}}^{t_{2}} P_{i n}^{H L S}(\tau)-P_{i n}(\tau) \mathrm{d} \tau,
$$

where the energy balance of the communications without HLS in wave variables using (3) and (2) yields

$$
\int_{t_{1}}^{t_{2}} P_{i n}(\tau) \mathrm{d} \tau=\frac{1}{2} \int_{t_{1}}^{t_{2}} u_{l}^{2}(\tau)-u_{r}^{2}(\tau)+v_{r}^{2}(\tau)-v_{l}^{2}(\tau) \mathrm{d} \tau
$$

This equation equally applies for HLS with the only difference that $u_{r}(t)$ is replaced by the distorted signal $u_{r}^{*}(t)=$ $u_{l}\left(t_{1}-T_{1}\right)$, which is constant for $t \in\left[t_{1}, t_{2}\right]$. Now (5) reduces to

$$
\begin{aligned}
E_{\text {in }}^{H L S}(t)-E_{\text {in }}(t) & =\int_{t_{1}}^{t_{2}}-u_{r}^{* 2}(\tau)+u_{r}^{2}(\tau) \mathrm{d} \tau \\
& =-u_{l}^{2}\left(t_{1}-T_{1}\right) \int_{t_{1}}^{t_{2}} \mathrm{~d} \tau+\int_{t_{1}}^{t_{2}} u_{r}^{2}(\tau) \mathrm{d} \tau
\end{aligned}
$$

with $u_{r}(\tau)=u_{l}\left(\tau-T_{1}\right)$. As the scattered communication line is lossless without HLS, clearly energy is generated if $E_{i n}^{H L S}(t)-E_{i n}(t)<0$ with this difference being exactly the amount of energy generated; the communication two-port is active, which proofs the result (4).

Corollary 1: If the lefthand side of (4) is larger than zero, then the communication two-port dissipates energy, hence is strictly passive.

Some more insight on the dependence on the signal behavior is provided by the following considerations.

Corollary 2: If

$$
\operatorname{sign}\left\{u_{l}\left(t_{1}-T_{1}\right)\right\} \cdot \operatorname{sign}\left\{\dot{u}_{l}\left(t-T_{1}\right)\right\}<0 \quad \forall t \in\left[t_{1}, t_{2}\right]
$$

holds with $\dot{u}_{l}$ the time derivative of $u_{l}$, then the communication two-port is active.

Proof: If (8) holds, then with the first order Taylor expansion of $u_{l}\left(t-T_{1}\right)$ around $t_{1}$ $u_{l}\left(t-T_{1}\right)=u_{r}(t)=u_{r}\left(t_{1}+\Delta t\right)=u_{r}\left(t_{1}\right)+\dot{u}_{r}\left(t_{1}+\sigma \Delta t\right)$ with $\sigma \in[0,1]$ the Lagrangian remainder

$$
\left(u_{r}\left(t_{1}\right)+\dot{u}_{r}\left(t_{1}+\sigma \Delta t\right)\right)^{2}<u_{r}\left(t_{1}\right)^{2} .
$$

If this holds $\forall \Delta t \in\left[0, t_{2}-t_{1}\right] \quad$ then $u_{l}\left(t-T_{1}\right)^{2}<u_{l}^{2}\left(t_{1}-T_{1}\right) \forall t \in\left[t_{1}, t_{2}\right]$, thus (4) is fulfilled; the two-port is active.

Corollary 3: With the same arguments and Corollary 1 it follows that if

$$
\operatorname{sign}\left\{u_{l}\left(t_{1}-T_{1}\right)\right\} \cdot \operatorname{sign}\left\{\dot{u}_{l}\left(t-T_{1}\right) \geq 0 \quad \forall t \in\left[t_{1}, t_{2}\right] .\right.
$$

holds, the communication two-port is passive.
Proposition 1: If the signal $u_{r}^{*}(t)=0$ is set to zero during packet loss passivity is preserved.

Proof: Clearly, $u_{r}^{* 2}(t)=0 \leq u_{l}^{2}\left(t-T_{1}\right)$ for $t \in\left[t_{1}, t_{2}\right]$ results in a overall positive term in the integral in (7) being sufficient for passivity.

Remark: These results equally apply to the backward path if $u_{l}\left(t-T_{1}\right)$ is replaced by $v_{r}\left(t-T_{2}\right)$ in (4), (8), (9) and Proposition 1.

Depending on the signal behavior we now have sufficient conditions for the passivity/non-passivity of the communication two-port. Note that the sufficiency is only given if HLS is applied in the time interval $\left[t_{1}, t_{2}\right]$ in the forward or backward path. If HLS occurs at the same time in the forward and the backward path, the possible energy generation might be compensated by energy dissipation.

Corollary 4: For a HLS occuring in the forward and the backward path in the same time interval $\left[t_{1}, t_{2}\right]$ with $t_{1} \geq \sup \left\{T_{1}, T_{2}\right\}$ the communication two-port is active, if

$-\left(t_{2}-t_{1}\right) \cdot\left(u_{l}^{2}\left(t_{1}-T_{1}\right)+v_{r}^{2}\left(t_{1}-T_{2}\right)\right)+\int_{t_{1}}^{t_{2}}\left(u_{r}^{2}+v_{l}^{2}\right)(\tau) \mathrm{d} \tau<0$.

Proof: This directly follows from (4), the foregoing remark and (6).

In summary: the HLS algorithm is potentially active, whereas the zeroing strategy is passive.

\section{B. Simulations}

The behavior of the HLS algorithm and the zeroing strategy is investigated in simulations. The HSI is modeled as a pure velocity source providing a sinusoidal HSI-velocity with a frequency of $2 \mathrm{rad} / \mathrm{s}$. The teleoperator is modeled with a mass of $m_{t}=0.23 \mathrm{~kg}$ and a damping coefficient of $b_{t}=0.04 \mathrm{~kg} / \mathrm{s}$. Furthermore a damper environment with $b_{e}=0.1 \mathrm{~kg} / \mathrm{s}$ is assumed. As time-varying delay either results in higher constant delay or in additional loss depending

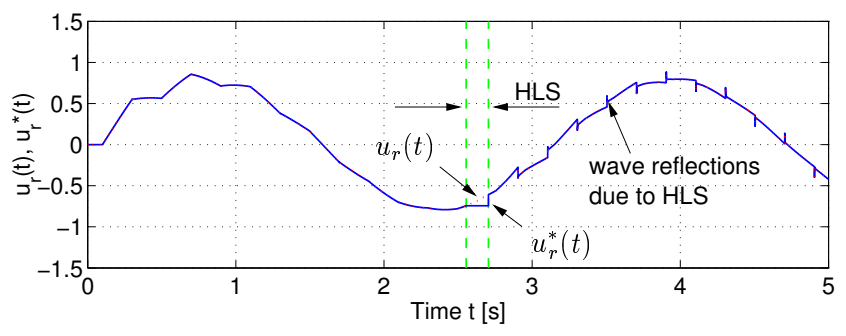

Fig. 4. Input and output signal of single applied HLS algorithm.

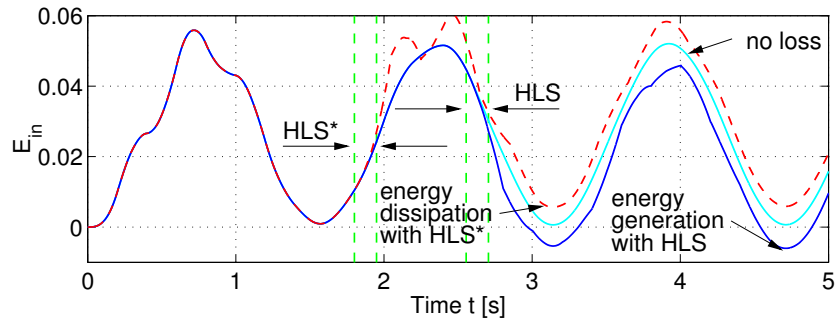

Fig. 5. Energy generated or dissipated HLS; in case of no HLS the network is lossless. 


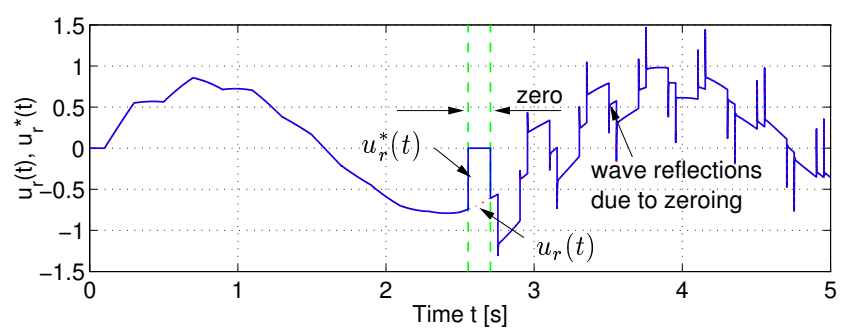

Fig. 6. Input and output signal when setting $u_{r}^{*}(t)=0$ during packet loss.

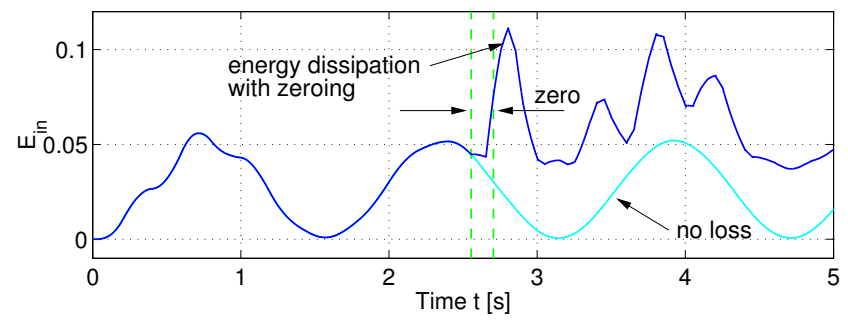

Fig. 7. Energy when setting $u_{r}^{*}(t)=0$ during packet loss; observe that this is strictly passive.

on the processing algorithm the delays in the forward and backward paths are kept constant $T_{1}=T_{2}=100 \mathrm{~ms}$, a signal outage occurs once for $150 \mathrm{~ms}$ at time $t=2.5 \mathrm{~s}$ in the forward path only.

The HLS algorithm distorts the signal as shown in Fig. 4, where the outgoing signal $u_{r}(t)$ before and after the HLS is depicted. The energy generated in the communication twoport by this single HLS can be seen in Fig. 5, where negative values correspond to non-passivity; energy is computed from (3). The passivity condition (1) is violated, validating the result from Corollary 2.

The strategy of setting $u_{r}^{*}=0$ from Proposition 1, the so called zeroing is simulated as the simplest possible packet loss strategy, see Fig. 6. The energy in the communication depicted in Fig. 7 shows the strict passivity of this approach. As a side effect of zeroing high frequencies are introduced depending on the development of $u_{r}(t)$ during the signal outage resulting in wave reflections, see Fig. 6. In a real system these wave reflections disturb the perception of the remote environment, i.e. deteriorate transparency. Damping in the teleoperator/environment or the HSI/human which is not considered here damp out the wave reflections. Another drawback with respect to the performance is that in free space motion a non-recoverable position drift between HSI and teleoperator may occur. In Fig. 8 the positions of the teleoperator for the no loss

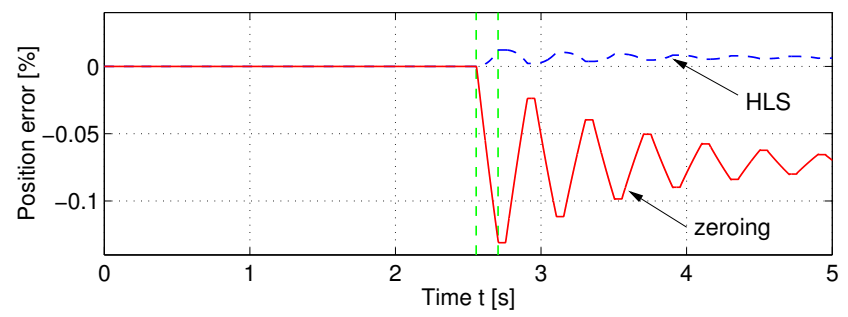

Fig. 8. Position error for zeroing strategy and HLS case and the zeroing strategy are compared. The position error $e_{t}(t)=1 / x_{\max }\left(x_{t, n o H L S}(t)-x_{t, \text { zeroing }}(t)\right)$ with the zeroing strategy shows high values of up to $13 \%$, whereas the position error induced by HLS is comparably low (1\%) In contact with a stiff environment the displayed force suddenly may break down due to zeroing.

\section{Passivation of Packet Processing}

On the basis of our results we propose two methodologies for the passive data recovery in case of packet loss in bilateral telepresence systems. As the buffering of packets itself has no effect on the passivity, it is not considered here.

\section{A. Bounded Rate}

The idea of the bounded rate approach is to estimate the energy input $u_{l}^{2}(t), v_{r}^{2}(t)$ to the communication two-port in order to appropriately bound the energy output $u_{r}^{* 2}(t), v_{l}^{* 2}(t)$ during data loss. This can be done seperately for the forward and the backward path, the following considerations are written down for the forward path only, but equally apply to the backward path. In order to estimate the energy input in this approach the rate of the transmitted signal is assumed to be bounded $|\dot{u}| \leq\left|\dot{u}_{\max }\right|$. In application this can be guaranteed by a rate limiter block just before the communication two-port. It is easy to show that this rate limiter does not generate energy, hence is passive.

Theorem 2: Considering data loss in the forward path within the time interval $\left[t_{1}, t_{2}\right]$ with the transmitted signal rate bounded by $|\dot{u}| \leq\left|\dot{u}_{\max }\right|$ the data recovery algorithm

$u_{r}^{*}(t)= \begin{cases}u_{r}\left(t_{1}\right)-\operatorname{sign}\left\{u_{r}\left(t_{1}\right)\right\} & \left|\dot{u}_{\max }\right| \Delta t \text { if } \operatorname{sign}\left\{u_{r}\left(t_{1}\right) u_{r}^{*}(t)\right\} \\ 0 & \text { otherwise }\end{cases}$

with $\Delta t \in\left[0, t_{2}-t_{1}\right]$ is passive.

Proof: For the passivity of the communication twoport including the data recovery for lost packets the energy balance must fulfill $E_{i n}^{*}(t) \geq 0$. As discussed before it is sufficient to show that $E_{i n}^{*}(t)-E_{\text {in }}(t) \geq 0$, where $E_{\text {in }}(t)$ represents the energy balance of the communication two-port without loss. The passivity of the zeroing strategy has been shown before, hence only the time interval $\left[t_{1}, t_{1}^{\prime}\right]$ until $u_{r}\left(t_{1}\right)$ and $u_{r}^{*}(t)$ take opposite signs has to be considered. For the energy balance to be positive $u_{r}^{2}(t) \geq u_{r}^{* 2}(t)$ must hold, see (7). Given the bounded rate the signal dynamics of the lost data is bounded by $\left|u_{r}\left(t_{1}+\Delta t\right)\right| \geq\left|u_{r}\left(t_{1}\right)\right|-\left|\dot{u}_{\max } \Delta t\right|$, hence $\left(u_{r}\left(t_{1}+\Delta t\right)\right)^{2} \geq\left(u_{r}\left(t_{1}\right)-\operatorname{sign}\left\{u_{r}\left(t_{1}\right)\right\}\left|\dot{u}_{\max }\right|\right)^{2}=u_{r}^{* 2}(t)$ according to the proposed method. Thus this algorithm passifies the communication two-port with data loss.

Simulations are performed under the same conditions as above with a rate limiter in the forward path just before the communications $\left(\dot{u}_{\max }=3\right)$ in order to guarantee the bounded rate. Fig. 9 shows the signal development before and after the application of this strategy. According to the energy balance in Fig. 10 the communication two-port shows strictly passive behavior, but is less conservative than the zeroing strategy. As a result the performance in 


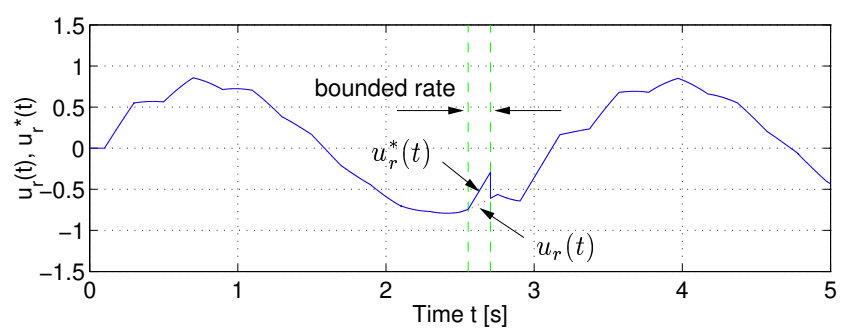

Fig. 9. Input and output signal when setting $u_{r}^{*}(t)$ to the bounded rate during packet loss.

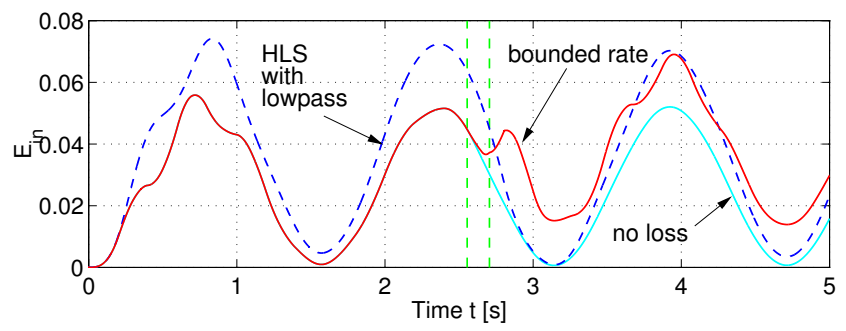

Fig. 10. Energy dissipated in communication two-port with bounded rate approach and HLS with low pass filter.

terms of position tracking is increased as shown in Fig. 11. The position error is with $2 \%$ far lower than the zeroing strategy (up to 13\%). Furthermore the wave reflections are reduced. In real applications especially during environment contact high rates of $v_{l}(t)$ may occur. These components are filtered out by this approach depending on the rate limit set; this has an impact on the perception of sudden changes in environment. Higher rate limits could prevent this, but then the performance becomes lower during loss.

\section{B. HLS with Low Pass Filtering}

A simple solution applied in a heuristic approach, see [15], is low pass filtering inside the communication twoport. The low pass is placed directly after the HLS packeting algorithm. High frequency components and wave reflections introduced by the HLS are filtered out; as a result the energy output of the communication two-port is reduced. A formal proof of passivity is difficult; a design rule for the low pass is not given here. For the special case in the simulations performed, see Fig. 10 a 1st order low pass filter with a bandwidth of $\omega_{0}=20 \mathrm{rad} / \mathrm{s}$ is appropriate for passivity. The position error in Fig. 11 with a maximum of $2.5 \%$ reveals good performance.

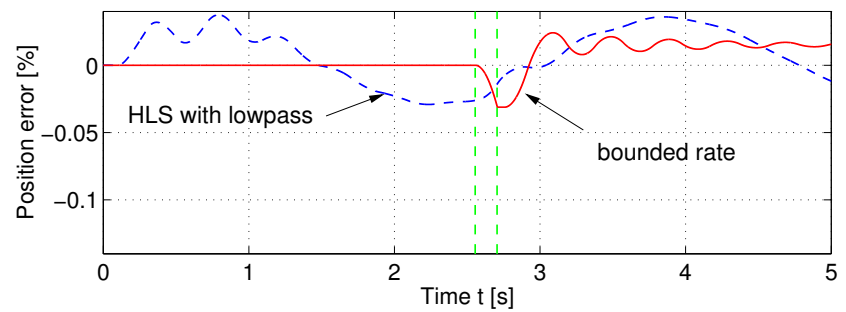

Fig. 11. Position error for bounded rate strategy and HLS with lowpass filtering

\section{CONCLUSIONS}

This paper discusses the problem of packet loss in a certain class of networked control systems, namely telepresence systems. The potentially destabilizing effect of packet loss in combination with the Hold Last Sample algorithm has been shown. A sufficient condition for HLS nonpassivity has been derived. Furthermore, a strictly passive strategy zeroing signals during packet loss and two other stabilization methods based on rate bounding and low pass filtering have been discussed.

\section{Acknowledgments}

The detailed comments by the anonymous reviewers are highly appreciated.

\section{REFERENCES}

[1] M. Buss and G. Schmidt, "Control Problems in Multi-Modal Telepresence Systems," in Advances in Control: Highlights of the 5th European Control Conference ECC'99 in Karlsruhe, Germany (P. Frank, ed.), pp. 65-101, Springer, 1999.

[2] R. Anderson and M. Spong, "Bilateral Control of Teleoperators with Time Delay," IEEE Transaction on Automatic Control, vol. 34, pp. 494501, 1989.

[3] R. Lozano, N. Chopra and M. Spong, "Passivation of Force Reflecting Bilateral Teleoperators with Time Varying Delay," in Proceedings of the 8. Mechatronics Forum, (Enschede, Netherlands), 2002.

[4] N. Chopra, M. W. Spong, S. Hirche, and M. Buss, "Bilateral Teleoperation over Internet: the Time Varying Delay Problem," in Proceedings of the American Control Conference, (Denver, CO), 2003.

[5] C. Secchi, S. Stramigioli and C. Fantuzi, "Digital Passive Geometric Manipulation," in Proceedings of the IEEE International Conference on Robotics and Automation ICRA'03, 2003, Taipei, Taiwan.

[6] J. Nilsson, "Real-time Control Systems with Delays," PhD thesis , Lund Institute of Tecnology, 1998.

[7] Q. Ling and M.D. Lemmon, "Robust Performance of Soft Real-time Networked Control Systems with Data Dropout," in Proceedings of the 41nd IEEE Conference on Decision and Control CDC'02, 2002, Las Vegas, US.

[8] B. Sinopoli, L. Schenato, M. Franceschetti, K. Poolla, M. I. Jordan and S.S. Sastry, "Kalman Filtering with Intermittent Observations," in Proceedings of the 42nd IEEE Conference on Decision and Control $C D C^{\prime} 03,2003$, Maui, Hawaii, US.

[9] P.L. Tang and C.W. de Silva "Ethernet-based Predictive Control of an Industrial Hydraulic Machine," in Proceedings of the 42nd IEEE Conference on Decision and Control CDC'03, 2003, Maui, Hawaii, US.

[10] P. Berestesky, N. Chopra and M. Spong "Discrete Time Passivity in Bilateral Teleoperation over the Internet," in Proceedings of the IEEE International Conference on Robotics and Automation ICRA'04, 2004, New Orleans, US.

[11] G. Niemeyer and J.-J. Slotine, "Stable Adaptive Teleoperation," IEEE Journal of Oceanic Engineering, vol. 16, pp. 152-162, January 1991.

[12] Y. Yokokohji and T. Yoshikawa, "Bilateral Control of MasterSlave Manipulators for Ideal Kinesthetic Coupling Formulation and Experiment," IEEE Transactions on Robotics and Automation, vol. 10, pp. 605-619, October 1994.

[13] Stramigioli, S., van der Schaft, A., Maschke, B. and Melchiorri, C., "Geometric Scattering in Robotic Telemanipulation." IEEE Transactions on Robotics and Automation 20, No.Y (July 2002).

[14] K. Kosuge and H. Murayama and K. Takeo, "Bilateral Feedback Control of Telemanipulators via Computer Network," in Proceedings of the IEEE/RSJ International Conference on Intelligent Robots and Systems IROS, 1996, Osaka, Japan, pp. 1380-1385.

[15] H. Baier, M. Buss, and G. Schmidt, "Stabilität und Modusumschaltung von Regelkreisen in Teleaktionssystemen," at-Automatisierungstechnik, vol. 48, pp. 51-59, Februar 2000. 